\title{
Design of a power amplifier circuit for a starch gelatinization power supply system
}

\author{
Minghui You, Xue Li, Qixiang Sun, Liping Yin, Shijun Li, Jingsheng Liu ${ }^{\text {a }}$ \\ Jilin Agricultural University, Changchun 130118, Jilin, China. \\ amhyou000@163.com
}

\begin{abstract}
This paper presented the analysis and design of a power amplifier circuit for a starch gelatinization power supply system. This circuit can realize some functions: High power, high efficiency, no distortion, good heat dissipation. In order to meeting the requirements of the load, it outputted a certain signal power, The higher efficiency was to refer to the power supply of the DC power supply was converted into the power of the useful signal, with the advantages of economic saving and more reliable performance. The circuit has been designed and made, including preamplifier, amplifier, filter circuit etc. This paper introduces the design process of combinational logic circuit and the working principle of the system.
\end{abstract}

Keywords: Power amplifier circuit; driving circuit; power supply system.

\section{Introduction}

In the gelatinization power supply system, the output stage of the circuit is required to output a certain amount of power to drive the load [1]. To meet this demand, we need to design a suitable power amplifier, which can amplify the original signal. Amplifier was the most basic functional circuit [2]. It converted small signals into large signals [3]. In accordance with the different requirements of the circuit output, the amplifier was divided into small signal power amplifiers and power amplifiers [4]. Small-signal amplifier actived devices in the linear range of work, Signal was not distorted.. Power amplifier was mainly to achieve high efficiency and high power output circuit [5]. The core was still the energy conversion circuit [6]. The higher efficiency was to refer to the power supply of the DC power supply is converted into the power of the useful signal, with the advantages of economic saving and more reliable performance [7, 8]. The power amplifier circuit should consider the problem of reducing the nonlinear distortion, the heat dissipation and the protection of the power amplifier tube [9].

\section{Overall System Framework}

\subsection{The principle of the power system}

In generally, the power supply system of the equipment was mainly composed of the driving circuit, power amplifier circuit, matching circuit, energy conversion device and feedback circuit. The principle of the power supply system of the power system was shown in fig. 1:

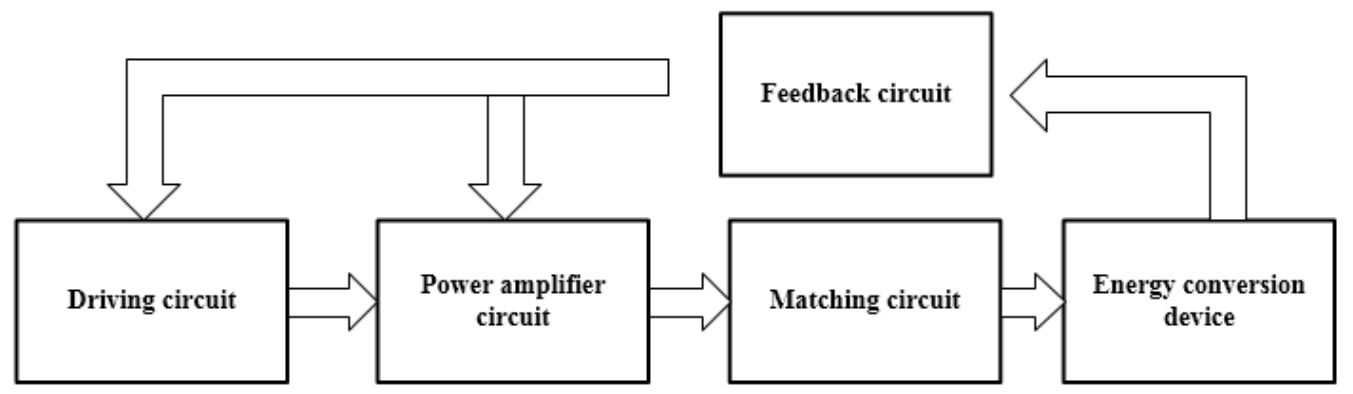

Fig. 1 The principle of the power system 


\subsection{Working Process}

(1) Driving circuit

Drive circuit generated the signal of a specific frequency to promote the work of the power amplifier. This signal can be a sinusoidal signal or a pulsed signal; the selected specific frequency is the transducer frequency. Currently, the frequencies used in system equipment are $10 \mathrm{kHz}, 20 \mathrm{kHz}, 30$ $\mathrm{kHz}, 40 \mathrm{kHz}$, and $100 \mathrm{kHz}$.

(2) Power amplifier circuit

Power amplifier was the core component of power, it has a variety of structural forms, class a power amplifier tube, class $\mathrm{AB}$ amplifier, $\mathrm{B}$ amplifier, switching amplifier. Power was generally ranging from $50 \mathrm{~W}$ to $5000 \mathrm{~W}$.

(3) Matching circuit

Impedance matching circuit was designed to make the output impedance of the generator match with the transducer, and the transducer converted the electric signal to the output of the energy.

(4) Feedback circuit.

Feedback circuit, mainly to provide two aspects of the feedback signal: the output power feedback signal and frequency feedback signal.

(5) Energy conversion device

The power output of the energy conversion device was converted to the output of the power output, and the normal function of the system was accomplished.

\section{Operating Principle}

Usually we studied the four aspects of the problem the output power of the amplifier circuit, efficiency, distortion and heat dissipation.

First, the output power was as large as possible. Because the greater the output power, when the small signal input, the linearity of the better, the distortion will be smaller.

Second, never has a circuit been as efficient and accurate as the power amplifier circuit. As the power amplifier circuit in the last stage of the amplifier, so often work in high voltage, high current circumstances, and the vast majority of the energy consumed in the power amplifier at this level, so the level of power consumption directly determine the level the power consumption of the entire circuit.

Third, the nonlinear distortion was studied theoretically. The results showed that the image distortion was smaller and the compaction ratio is greater. As the power amplifier circuit to output as much power as possible, then the device was often used in extreme operating conditions, the transistor would inevitably appear nonlinear distortion, and how to reduce this distortion was the key to our facing problem.

Fourth, heat radiation was the crux of all key technologies. As the power amplifier circuit for a long time in high-power state, so the circuit would heat, improper burned the power tube, so a good power amplifier circuit, there must be a good heat dissipation. 


\section{Circuit Design}

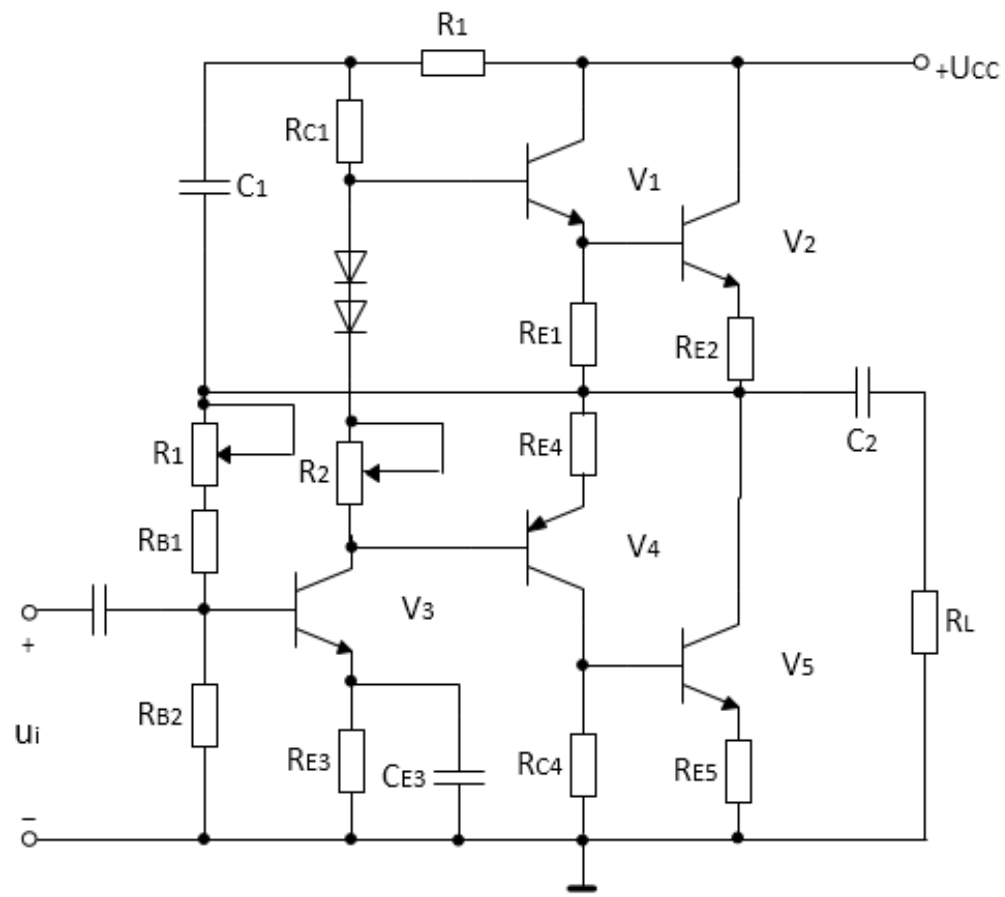

Fig. 2 Power amplifier circuit diagram

Parameter calculation:

$$
\begin{aligned}
& P_{o}=V_{O} I_{O} \\
& P_{o}=V_{O} I_{O}=\frac{V_{o M}}{\sqrt{2}} \bullet \frac{I_{o M}}{\sqrt{2}} \\
& I_{O M}=V_{O M} / R_{L} \\
& P_{o}=V_{O} I_{O}=\frac{V_{o M}}{\sqrt{2}} \bullet \frac{V_{o M}}{\sqrt{2} R_{L}}=\frac{1}{2} \bullet \frac{V_{o M}{ }^{2}}{R_{L}} \\
& P_{o M}=\frac{1}{2} \bullet \frac{U_{C C}^{2}}{R_{L}} \\
& P_{T}=2 P_{T 1}=\frac{2}{R_{L}}\left(\frac{V_{o m} U c C}{\pi}-\frac{V_{o m}^{2}}{4}\right) \\
& \eta_{\max } \approx 78.5 \%
\end{aligned}
$$

According to the verification of the actual circuit, the design of the control circuit was able to complete its function.

\section{Summary}

The power amplifier circuit were designed to determine the program, qualitative analysis, and then calculate the parameter values, welding would be measured after the success of debugging. Until it reached the expected design requirements, and then batched production. To achieve better results, setting the practice can be divided into the design process analysis of the above steps.

\section{Acknowledgments}

The authors wish to express their gratitude to the projects: Jilin Province Education Department Project (No.2015Y174), Jilin Agricultural University learning program, Jilin Provincial Department of human resources and social security Project (No. 2015Y24) for their generous support of this work. 


\section{References}

[1] Cheng Zhengwu, Cheng Dabei. Reliability Design for Low Frequence Power Amplifiers [J]

[2] Journal of Southern Yangtze University (Natural Science Edition), 2013, 2:483-486.

[3] Cui KejiResearch on High Efficiency Power Amplifier [D] Fudan University, 2013, 6-20.

[4] Gao Youfu, a Summary of Transistor Power Amplification circuit [J] Journal of Changjing Engineering Engineering Vocational College, 2005, 22:42-47.

[5] Xia Wei ,Design and Realization of a kind of Class AB Power Amplifier with Signal Source [D]

[6] Harbin Engineering University, 2013, 21-29.

[7] Lin Yujia, The design of a high-performance audio power amplifier [D], Liaoning University, $2013,12-24$

[8] Shi Xinfeng, Analysis and design of double input direct current with holohedral symmetry and whole complementry [J], Journal of Henan Mechanical and Electrical Engineering College, 2004, 12:53-54.

[9] Yang Jiansong, Design and Thermal Analysis of the Ka-band Power Amplifier based GaN [D], Beijing Institute of Technology, 2015, 3-18.

[10] Li Weiwen, Discussing of Transistor Power Amplification Circuit [J] Journal of Hunan Industry Polytechnic, 2008, 8:20-22.

[11]Zhang Yue, Design and research of class AB power amplifier, [D] GuiZhou University, 2011, $22-31$. 